\title{
Dynamic change of neutrophil to lymphocyte ratio and hemorrhagic transformation after thrombolysis in stroke
}

\author{
Zhiliang Guo ${ }^{1,2 \dagger}$, Shuhong Yu ${ }^{1 \dagger}$, Lulu Xiao ${ }^{1 \dagger}$, Xin Chen ${ }^{1}$, Ruidong Ye ${ }^{1}$, Ping Zheng ${ }^{3}$, Qiliang Dai ${ }^{1}$, Wen Sun ${ }^{1}$, \\ Changsheng Zhou ${ }^{4}$, Shuiping Wang ${ }^{5}$, Wusheng Zhu ${ }^{{ }^{*}}$ and Xinfeng Liu ${ }^{1 *}$
}

\begin{abstract}
Background: The neutrophil to lymphocyte ratio (NLR) has been shown to predict short- and long-term outcomes in ischemic stroke patients. We sought to explore the temporal profile of the plasma NLR in stroke patients treated with intravenous thrombolysis (IVT) and its relationship with intracranial bleeding complications after thrombolysis.

Methods: A total of 189 ischemic stroke patients were prospectively enrolled. Blood samples for leukocyte, neutrophil, and lymphocyte counts were obtained at admission and at 3-6, 12-18, and 36-48 h after IVT. Head CT was performed on admission and repeated after 36-48 h, and a CT scan was done immediately in case of clinical worsening. Hemorrhagic events were categorized as symptomatic intracranial hemorrhage $(\mathrm{s} \mid \mathrm{CH})$ and parenchymal hematomas (PH) according to previously published criteria.

Results: An increasing trend in the NLR was observed after stroke, and the NLR was higher in patients who developed PH or sICH at 3-6, 12-18, and 36-48 h after IVT $(P<0.01)$ than in those without PH or sICH. The optimal cutoff value for the serum NLR as an indicator for auxiliary diagnosis of PH and sICH was 10.59 at 12-18 h. Furthermore, the NLR obtained at 12-18-h post-treatment was independently associated with PH (adjusted odds ratio [OR] 1.14) and sICH (adjusted OR 1.14). In addition, patients with a NLR $\geq 10.59$ had an 8.50-fold greater risk for $\mathrm{PH}(95 \%$ confidence interval [Cl] 2.69-26.89) and a 7.93-fold greater risk for slCH (95 \% Cl 2.25-27.99) than patients with a NLR $<10.59$.
\end{abstract}

Conclusions: NLR is a dynamic variable, and its variation is associated with HT after thrombolysis in stroke patients.

Keywords: Neutrophil to lymphocyte ratio (NLR), Ischemic stroke, Thrombolysis, Hemorrhagic transformation, Biomarker

Abbreviations: AUC, Area under the curve; BBB, Blood-brain barrier; Cl, Confidence interval; DBP, Diastolic blood pressure; HT, Hemorrhagic transformation; I/R, Ischemia/reperfusion; INR, International normalized ratio; IQR, Interquartile range; IV rtPA, Intravenous recombinant tissue plasminogen activator; IVT, Intravenous thrombolysis; MMP-9, Matrix metalloproteinase-9; NETs, Neutrophil extracellular traps; NIHSS, National Institutes of Health Stroke Scale; NLR, Neutrophil to lymphocyte ratio; OR, Odds ratio; PH, Parenchymal hematoma; ROC, Receiver operating characteristic; ROS, Reactive oxygen species; SBP, Systolic blood pressure; SD, Standard deviation; sICH, Symptomatic intracerebral hemorrhage

\footnotetext{
*Correspondence: zwsemail@sina.com; xfliu2@vip.163.com

${ }^{\dagger}$ Equal contributors

${ }^{1}$ Department of Neurology, Jinling Hospital, Medical School of Nanjing

University, 305 E Zhongshan Rd, Nanjing 210002, Jiangsu Province, China

Full list of author information is available at the end of the article
} 


\section{Background}

Multiple randomized controlled trials have demonstrated the efficacy of intravenous recombinant tissue plasminogen activator (IV rtPA) administered up to $4.5 \mathrm{~h}$ after the onset of symptoms of ischemic stroke [1]. However, the risk of hemorrhagic transformation (HT) is increased by as much as tenfold after IV rtPA, largely due to reperfusion injury and the toxic effects of rtPA [2]. In addition to the already known indicators for HT [2], the detection of new paradigms is still worthwhile. In addition, an improved understanding of the prevention or early risk assessment of rtPA-related HT may also be applicable to other reperfusion strategies such as endovascular therapy.

In animal studies, neutrophils have been shown to contribute to intracerebral hemorrhaging after treatment with rtPA following cerebral ischemia, while depletion of neutrophils reduces blood-brain barrier (BBB) disruption and the rate of HT $[3,4]$. In humans, infiltration of matrix metalloproteinase-9 (MMP-9)-positive neutrophils is associated with BBB breakdown, basal lamina type IV collagen degradation, and HT [5]. Recent studies suggested that the initial neutrophil to lymphocyte ratio (NLR) is associated with mortality and infarct size in ischemic stroke $[6,7]$ and can predict the 90-day outcome after endovascular therapy [8]. However, all of these studies mainly focused on static NLR values at baseline, which may not reflect the comprehensive dynamic changes of patients' conditions. Furthermore, there is also a lack of information on the clinical value of the NLR in acute ischemic stroke patients treated with IV rtPA, especially its relationship with the most serious and common complication of IV rtPA treatment, HT.

Thus, we aimed to explore the temporal variation of the NLR in patients and its relationship with the most serious subtypes of HT, namely symptomatic intracranial hemorrhage $(\mathrm{sICH})$ and parenchymal hematoma $(\mathrm{PH})$, in patients with ischemic stroke treated with IV rtPA [9].

\section{Methods}

\section{Study population}

Consecutive ischemic stroke patients admitted to the Departments of Neurology at two hospitals (Jinling Hospital and PLA 123 Hospital, both large comprehensive hospitals) from March 2012 to August 2015 were prospectively recruited. The inclusion criteria for enrollment were (1) age $\geq 18$ years and (2) diagnosis of acute ischemic stroke and treatment with IV rtPA within 4.5-h post-onset. The study exclusion criteria were (1) evidence of active infection before admission or any systemic infection that occurred during the first $48 \mathrm{~h}$ after treatment with IV rtPA (41 patients); (2) cancer, chronic inflammation, autoimmune disease, or steroid therapy (6 patients); and (3) unavailability to complete blood cell count or medical records ( 8 patients discharged on the same day of admission). At last, 189 consecutive ischemic stroke patients were included in the current study. The study protocol was approved by the Institutional Human Research Ethics Committees of Jinling Hospital and PLA 123 Hospital, and all patients or their relatives gave informed consent.

\section{Treatment administration}

IV rtPA (alteplase, $0.9 \mathrm{mg} / \mathrm{kg}$ up to a maximum of $90 \mathrm{mg} / \mathrm{kg}$ ) was used with $10 \%$ of the total dosage as a bolus, followed by a 60 -min infusion of the remaining dose. Patients who were receiving a bridging therapy consisting of IV rtPA followed by endovascular therapy were also enrolled. The method of endovascular therapy, such as local intra-arterial thrombolysis using rtPA, mechanical thrombectomy, angioplasty, stent placement, or multimodal endovascular therapy, was left to the discretion of the neurointerventionists.

\section{Clinical protocol and laboratory tests}

Patient's medical history, including potential stroke risk factors, clinical examination findings, blood and coagulation test results, 12-lead electrocardiographs, and chest radiographs were obtained at admission. Stroke severity was assessed by a certified neurologist using the National Institutes of Health Stroke Scale (NIHSS) at admission and at 3-6, 12-18, and 36-48 h after treatment with IV rtPA. Neurological deterioration was defined as death or an increase of $\geq 4$ points in the NIHSS score between the two examinations [10].

Venous blood samples were obtained from all patients at admission and at 3-6, 12-18, and 36-48 h after treatment with IV rtPA. Total leukocyte, neutrophil, and lymphocyte counts were determined using a COULTER LH780 Hematology Analyzer (Beckman Coulter, Inc, Orange County, CA). The NLR was calculated as the ratio of the percentage of neutrophils over the percentage of lymphocytes, both obtained from the same blood sample.

\section{CT and intracranial hemorrhage}

On admission, all patients underwent a $\mathrm{CT}$ scan within the first $4.5 \mathrm{~h}$ of stroke onset. CT was repeated at 36$48 \mathrm{~h}$, and another CT scan was done immediately in case of rapid neurological deterioration to evaluate the presence of HT. CT images were reviewed by a neuroradiologist with extensive experience in acute stroke who was blinded to patients' medical records. PH was defined as hemorrhage with a mass effect according to previously published criteria [11]. sICH was defined as any hemorrhage in the brain on the CT scan accompanied by the presence of neurological deterioration [10]. 


\section{Statistical analysis}

To compare baseline characteristics between groups according to the presence of $\mathrm{PH}$ or $\mathrm{sICH}$, parametric and non-parametric comparisons were performed with the $t$ test, $\chi^{2}$ test, and Mann-Whitney $U$ test as appropriate. The relation of the NLR with two endpoints was investigated using logistic regression models. For multivariate analysis, we first included age and sex (model 1) and then additionally included variables that significantly correlated with $\mathrm{PH}$ or $\mathrm{sICH}$ in the univariate analysis $(P<0.10$; models 2 or 3$)$. Receiver operating characteristic (ROC) curves were used to test the overall discriminative ability of the NLR for PH or $\mathrm{sICH}$ and to establish optimal cutoff points at which the sum of the specificity and sensitivity was the highest. The differences in discriminative ability were tested using the DeLong method [12]. Finally, logistic regression analysis was performed again with the same independent variables as in the previous model, except for values of the NLR that were included as a binary variable according to the cutoff point. Statistical analysis was performed using SPSS for Windows, version 17.0 (SPSS Inc., Chicago, IL, USA) and SAS version 9.1 (SAS Institute Inc., Cary, NC). Two-tailed significance values were applied, and statistical significance was defined as $P<0.05$.

\section{Results}

Baseline characteristics of patients

One hundred eighty-nine patients with ischemic stroke met the study criteria. The demographic and clinical characteristics between the included and excluded patients are detailed in Additional file 1: Table S1. These included cohorts from different hospitals are described in Additional file 1: Table S2. Among the 189 patients, 28 (14.8\%) presented with $\mathrm{PH}$, and 17 (9.0 \%) developed $\mathrm{sICH}$. The mean time of sICH (determined by head CT) was $12.23 \pm 7.74 \mathrm{~h}$ after thrombolysis. The main baseline characteristics of patients according to the presence or absence of $\mathrm{PH}$ or to the presence or absence of sICH are presented in Table 1.

\section{Temporal profile of NLR depending on the type of HT}

The NLR was obtained at four different time points: at admission, 3-6 h after rtPA treatment, 12-18 h after rtPA treatment, and 36-48 h after rtPA treatment. The temporal profiles of the NLR according to the presence of $\mathrm{PH}$ or sICH are presented in Figs. 1 and 2 and Additional file 1: Table S3 and Figure S1. The NLR at admission did not differ between patients with and without PH $(P=0.819)$. Thereafter, an increasing trend in the NLR was observed in both groups (Additional file 1: Figure S1). However, the NLR values in the PH group were significantly higher than

Table 1 Baseline characteristics of patients according to the presence/absence of $\mathrm{PH}$ or slCH

\begin{tabular}{|c|c|c|c|c|c|c|}
\hline & No PH $(n=161)$ & $\mathrm{PH}(n=28)$ & $P$ & No $\mathrm{slCH}(n=172)$ & $\mathrm{sICH}(n=17)$ & $P$ \\
\hline Age, years, mean (SD) & $64.1 \pm 10.3$ & $70.1 \pm 10.5$ & 0.005 & $64.6 \pm 10.4$ & $68.7 \pm 11.7$ & 0.133 \\
\hline Females, \% & $59(36.6)$ & $7(25.0)$ & 0.233 & $60(34.9)$ & $6(35.3)$ & 0.973 \\
\hline Body mass index, $\mathrm{kg} / \mathrm{m}^{2}$, mean (SD) & $24.4 \pm 3.1$ & $23.9 \pm 3.0$ & 0.401 & $24.4 \pm 3.0$ & $24.0 \pm 3.5$ & 0.601 \\
\hline Hypertension, \% & $101(62.7)$ & $21(75.0)$ & 0.210 & $111(64.5)$ & $11(64.7)$ & 0.989 \\
\hline Diabetes, \% & $50(31.1)$ & $7(25.0)$ & 0.519 & $51(29.7)$ & $6(35.3)$ & 0.629 \\
\hline Hyperlipidemia, \% & $73(45.3)$ & $12(42.9)$ & 0.807 & $79(45.9)$ & $6(35.3)$ & 0.400 \\
\hline Previous stroke, \% & $14(8.7)$ & $5(17.9)$ & 0.251 & $17(9.9)$ & $2(11.8)$ & 1.000 \\
\hline Coronary artery disease, $\%$ & $18(11.2)$ & $5(17.9)$ & 0.494 & $19(11.0)$ & $4(23.5)$ & 0.266 \\
\hline Atrial fibrillation, \% & $48(29.8)$ & $12(42.9)$ & 0.171 & $50(29.1)$ & $10(58.8)$ & 0.012 \\
\hline Current smokers, $\%$ & $53(32.9)$ & $8(28.6)$ & 0.650 & $56(32.6)$ & $5(29.4)$ & 0.791 \\
\hline Ongoing antiplatelet therapy, \% & $9(5.6)$ & $7(25.0)$ & 0.002 & $12(7.0)$ & $4(23.5)$ & 0.060 \\
\hline $\mathrm{SBP}, \mathrm{mm} \mathrm{Hg}$, mean (SD) & $148.4 \pm 18.4$ & $154.5 \pm 17.1$ & 0.104 & $148.9 \pm 18.2$ & $153.2 \pm 18.7$ & 0.302 \\
\hline DBP, mm Hg, mean (SD) & $81.4 \pm 9.8$ & $83.2 \pm 10.4$ & 0.368 & $81.3 \pm 9.7$ & $84.8 \pm 11.2$ & 0.163 \\
\hline Blood glucose, mmol/L, median (IQR) & $7.2(5.3-9.0)$ & $7.0(5.8-9.5)$ & 0.133 & $7.0(5.3-9.0)$ & $8.0(5.5-9.5)$ & 0.645 \\
\hline Platelets, $10^{9} / \mathrm{L}$, mean (SD) & $187.9 \pm 50.8$ & $178.9 \pm 40.0$ & 0.371 & $187.3 \pm 49.9$ & $179.7 \pm 44.5$ & 0.545 \\
\hline INR, mean (SD) & $1.01 \pm 0.08$ & $1.01 \pm 0.09$ & 0.468 & $1.01 \pm 0.08$ & $1.03 \pm 0.08$ & 0.302 \\
\hline Baseline NIHSS, median (IQR) & $11(6-15)$ & $17(10-22.5)$ & 0.001 & $12(6-15)$ & $13(10-20)$ & 0.198 \\
\hline Onset to treatment, min, mean (SD) & $170.6 \pm 48.8$ & $183.4 \pm 51.9$ & 0.207 & $171.1 \pm 48.9$ & $187.2 \pm 52.1$ & 0.198 \\
\hline IV rtPA + endovascular therapy, \% & $49(30.4)$ & $9(32.1)$ & 0.856 & $51(29.7)$ & $7(41.2)$ & 0.326 \\
\hline
\end{tabular}

DBP diastolic blood pressure, INR international normalized ratio, IQR interquartile range, $I V$ rtPA intravenous recombinant tissue plasminogen activator, NIHSS National Institutes of Health Stroke Scale, $P H$ parenchymal hemorrhage, SBP systolic blood pressure, SD standard deviation, s/CH symptomatic intracranial hemorrhage 


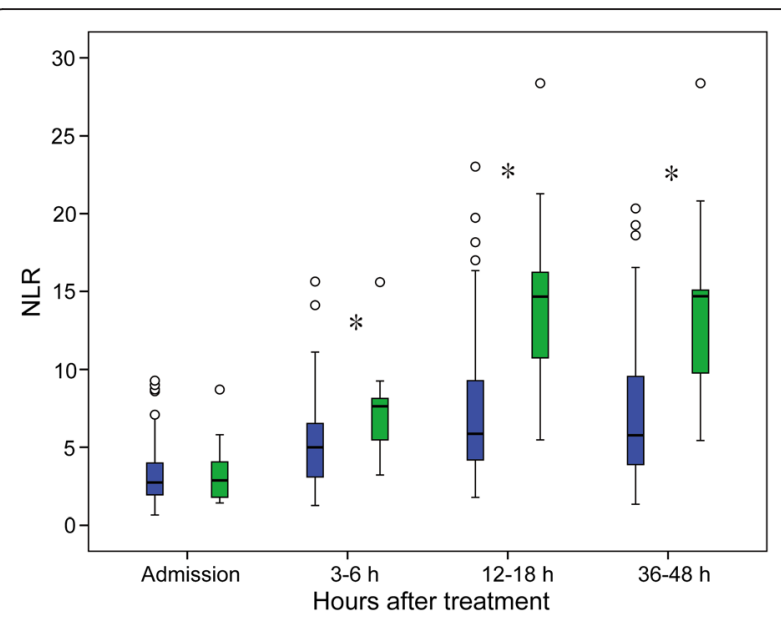

Fig. 1 Temporal profile of plasma neutrophil to lymphocyte ratio $(\mathrm{NLR})$ in stroke patients treated with recombinant tissue plasminogen activator (rtPA) according to the presence of parenchymal hemorrhage (PH). Blue boxes patients without $\mathrm{PH}$, green boxes patients with $\mathrm{PH}$. ${ }^{*} P<0.001$ between patients with and without $\mathrm{PH}$

those in the No PH group at 3-6, 12-18, and 36-48 h after rtPA $(P<0.001)$. The temporal profile of the NLR according to the presence of $\mathrm{sICH}$ was similar to that according to the presence of $\mathrm{PH}$.

\section{Association of plasma NLR with hemorrhagic transformation}

ROC curve analysis was performed to assess the best cutoff value of the NLR for discriminating $\mathrm{PH}$ and $\mathrm{sICH}$ (Fig. 3 and Table 2). The optimal cutoff value of the NLR that best distinguished the presence/absence of $\mathrm{PH}$

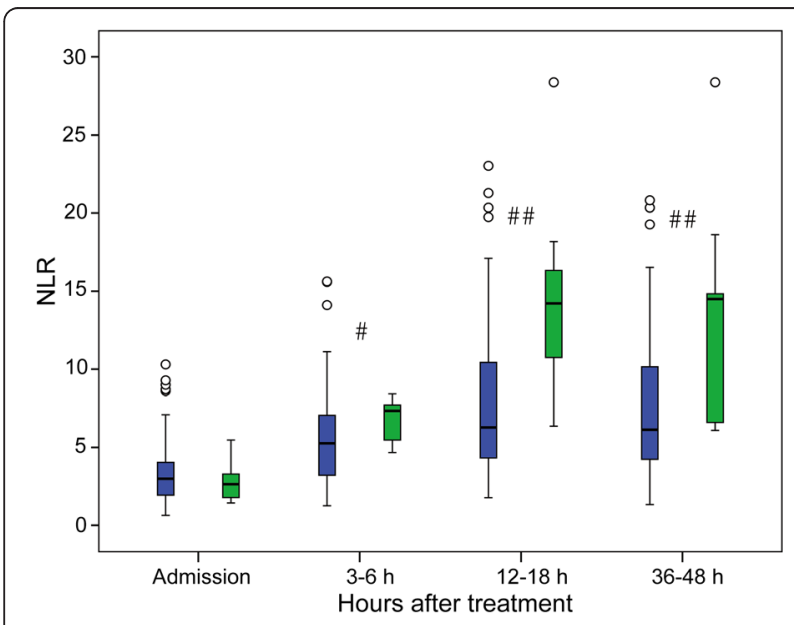

Fig. 2 Temporal profile of plasma neutrophil to lymphocyte ratio (NLR) in stroke patients treated with recombinant tissue plasminogen activator (rtPA) according to the presence of symptomatic intracranial hemorrhage $(\mathrm{s} \mid \mathrm{CH})$. Blue boxes patients without sICH, green boxes patients with $\mathrm{sICH} .{ }^{\#} P=0.009$ between patients with and without $\mathrm{SICH}$; ${ }^{\#} P<0.001$ between patients with and without sICH and $\mathrm{sICH}$ was 10.59 at $12-18 \mathrm{~h}$ after rtPA treatment, which can be obtained earlier than the NLR at 36-48 h after rtPA. The areas under the curve (AUCs) for the ability of the NLR to predict $\mathrm{PH}$ or $\mathrm{sICH}$ were 0.833 with $78.6 \%$ sensitivity and $79.5 \%$ specificity and 0.814 with $76.5 \%$ sensitivity and $75.6 \%$ specificity, respectively.

Table 3 summarizes the results of the binary logistic regression analysis of $\mathrm{PH}$ and $\mathrm{sICH}$. The NLR as a continuous variable was independently associated with a greater risk of $\mathrm{PH}$ with an adjusted odds ratio (OR) of 1.17 (95\% CI, 1.10-1.26) with adjustment for age and sex (model 1) and 1.14 (1.05-1.23) with further adjustment for ongoing antiplatelet therapy and baseline NIHSS (model 2), respectively. In addition, age (adjusted OR 1.06, $95 \% \mathrm{CI}$ : 1.01-1.11; $P=0.014$ ) and ongoing antiplatelet therapy (adjusted OR 4.00, 95 \% CI: 1.13$14.14 ; P=0.031)$ remained significant outcome predictors for $\mathrm{PH}$. Furthermore, in our study, the risk of $\mathrm{PH}$ was also associated with NLR levels as a dichotomous variable (OR 8.50, 95 \% CI: 2.69-26.89; $P<0.001$ ) after adjustment for age, sex, ongoing antiplatelet therapy, and baseline NIHSS (model 2). This relationship was further confirmed in the dose-response model (Additional file 1: Figure S2). For the binary logistic regression analysis for $\mathrm{sICH}$, similar associations were found between the NLR and sICH. A NLR level $\geq 10.59$ (OR 7.93, $95 \%$ CI $2.25-27.99 ; P=0.001)$ remained significantly associated with sICH after adjusting for age, sex, atrial fibrillation, and ongoing antiplatelet therapy (model 3).

\section{Discussion}

This study first shows that the NLR is a dynamic variable, and its variation is associated with HT after treatment with IV rtPA in patients with acute stroke. In addition, the best discriminating value of the NLR for $\mathrm{PH}$ and $\mathrm{sICH}$ was 10.59 or more at $12-18-\mathrm{h}$ posttreatment, which was associated with an 8.50-fold increased risk for $\mathrm{PH}$ and a 7.93-fold increased risk for sICH.

In previous studies, a high NLR was found to be independently associated with an increased risk of stroke in atrial fibrillation [13]. Moreover, the initial NLR is associated with infarct size and mortality rate in ischemic stroke [6, 7], and it also has predictive value for 90-day outcome after endovascular therapy [8]. However, the previous studies did not explore the clinical value of the NLR in acute ischemic stroke patients treated with IV rtPA.

Recently, Maestrini et al. reported that higher neutrophil counts and NLR before thrombolysis for cerebral ischemia are independently associated with $\mathrm{sICH}$ and worse outcome at 3 months [14]. However, they did not exclude patients with previous infections, which might contribute to the difference in the predictive values of 

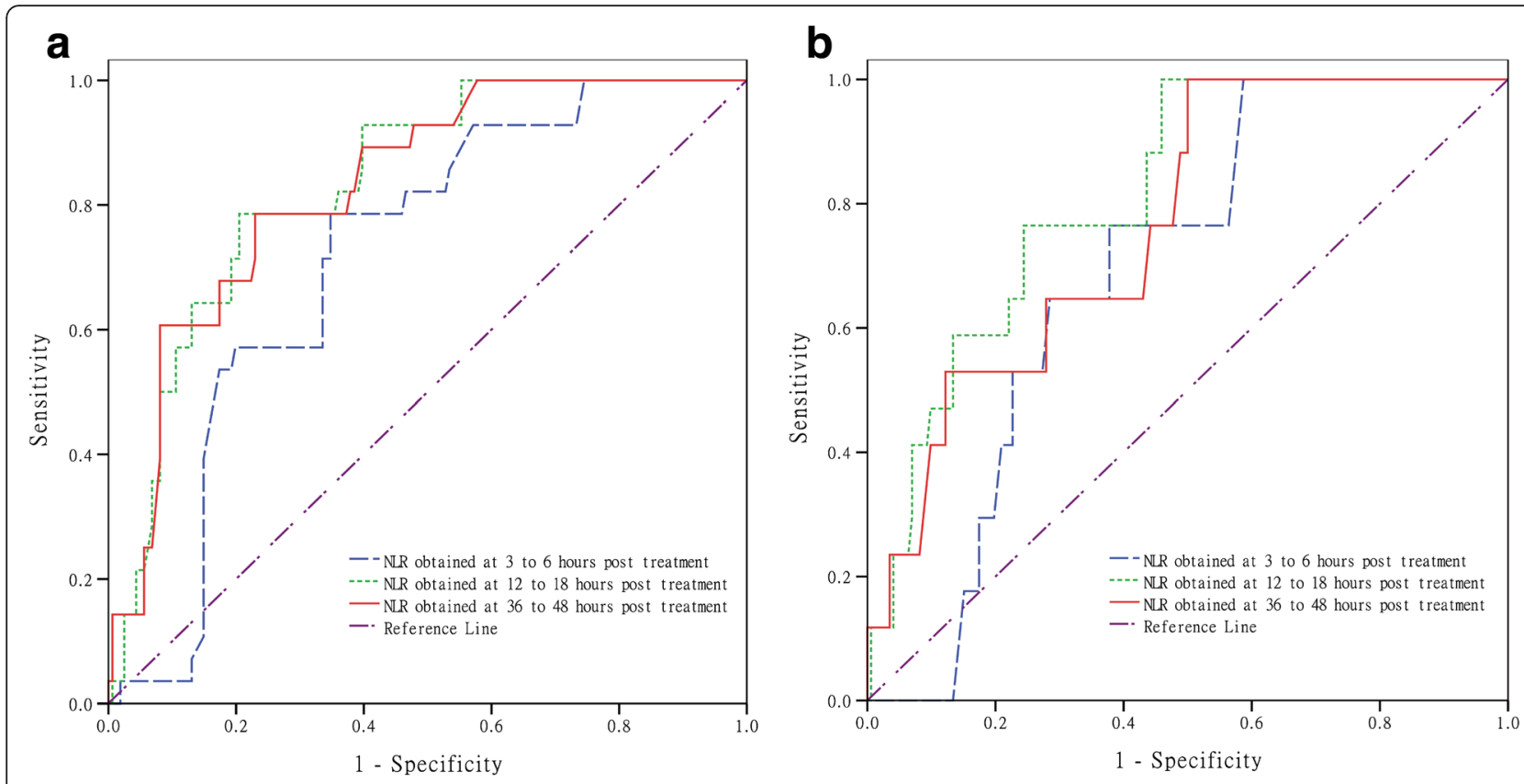

Fig. 3 Discriminative ability of the neutrophil to lymphocyte ratio (NLR) for parenchymal hemorrhage (PH) and symptomatic intracranial hemorrhage ( $\mathrm{S} \mathrm{CH})$. a Receiver operator characteristic (ROC) curve for NLR in auxiliary diagnosis of PH and $\mathbf{b}$ ROC curve for NLR in the auxiliary diagnosis of $\mathrm{slCH}$

the baseline NLR for sICH between their study and ours. That is because infections can lead to poor outcome after stroke via many different mechanisms, which may include (1) increased BBB disruption and tissue damage by neutrophil-derived various proteases, reactive oxygen species (ROS), as well as numerous inflammatory mediators; (2) impaired tissue reperfusion through endotheliadependent mechanisms; (3) increased platelet activation and microvascular coagulation; and (4) CRP-induced ischemic tissue injury via a complement-dependent mechanism $[15,16]$. Therefore, this shows that neutrophils are just one of many different mechanisms, and the association between the baseline NLR and endpoints may disappear after adjustment for infections. The reasons for such a difference may also relate to what the NLR represents. Changes observed in the baseline NLR could reflect the disease itself or external environment factors such as infection or cancer. Our results and their conclusions do not contradict per se because their study population is different to ours, with the NLR at baseline reflecting both the disease itself and the external environment factors in their study, but only the disease itself in our study.

The study by Maestrini et al. has a higher statistical power than our study based on the larger sample size [14], and we also believe that their results are fully credible and reliable. Higher numbers of baseline neutrophils at baseline have greater potential to induce tissue damage via the release of various proteolytic enzymes, ROS, and numerous inflammatory mediators [2]. Thus, in theory, patients with infection or other conditions that can potentially change baseline the NLR have a higher

Table 2 Diagnostic values of the neutrophil to lymphocyte ratio (NLR) for $\mathrm{PH}$ and $\mathrm{sICH}$

\begin{tabular}{|c|c|c|c|c|c|}
\hline & AUC (95 \% Cl) & $P$ & Cutoff value & Sensitivity (\%) & Specificity (\%) \\
\hline \multicolumn{6}{|l|}{ For PH } \\
\hline $3-6$ h NLR & $0.717(0.630-0.803)$ & $<0.001$ & 5.45 & 78.6 & 65.2 \\
\hline 12-18 h NLR & $0.833(0.764-0.903)$ & Reference & 10.59 & 78.6 & 79.5 \\
\hline 36-48 h NLR & $0.830(0.758-0.902)$ & 0.887 & 14.36 & 60.7 & 91.9 \\
\hline \multicolumn{6}{|l|}{ For sICH } \\
\hline $3-6$ h NLR & $0.691(0.597-0.785)$ & $<0.001$ & 5.45 & 76.5 & 62.8 \\
\hline $12-18$ h NLR & $0.814(0.728-0.900)$ & Reference & 10.59 & 76.5 & 75.6 \\
\hline 36-48 h NLR & $0.766(0.666-0.866)$ & 0.166 & 14.36 & 52.9 & 87.8 \\
\hline
\end{tabular}


Table 3 Associations of the neutrophil to lymphocyte ratio (NLR) with $\mathrm{PH}$ and $\mathrm{SICH}$

\begin{tabular}{|c|c|c|c|c|c|c|}
\hline & \multicolumn{3}{|l|}{ PH OR (95\% Cl) } & \multicolumn{3}{|l|}{ sICH OR (95\% Cl) } \\
\hline & Univariate analysis & Model 1 & Model 2 & Univariate analysis & Model 1 & Model 3 \\
\hline$\overline{N L R^{a}}$ & $1.19(1.10-1.28)^{*}$ & $1.17(1.10-1.26)^{*}$ & $1.14(1.05-1.23)^{* *}$ & $1.16(1.08-1.26)^{*}$ & $1.16(1.07-1.25)^{*}$ & $1.14(1.06-1.23)^{* *}$ \\
\hline $\operatorname{NLR}^{\mathrm{b}}(\geq 10.59$ vs $<10.59)$ & $14.22(5.34-37.91)^{*}$ & $11.86(4.35-32.36)^{*}$ & $8.50(2.69-26.89)^{*}$ & $10.06(3.11-32.52)^{*}$ & $9.55(2.84-32.04)^{*}$ & $7.93(2.25-27.99)^{*}$ \\
\hline
\end{tabular}

Model 1 bivariate logistic regression analyses with adjustment for age and sex. Model 2 bivariate logistic regression analyses with adjustment for age, sex, ongoing antiplatelet therapy, and baseline NIHSS. Model 3 bivariate logistic regression analyses with adjustment for age, sex, atrial fibrillation, and ongoing antiplatelet therapy. $\mathrm{Cl}$ confidence interval, $\mathrm{OR}$ odds ratio, $\mathrm{PH}$ parenchymal hemorrhage, $s / \mathrm{CH}$ symptomatic intracranial hemorrhage

${ }^{*} P<0.001 ;{ }^{* *} P<0.05$

${ }^{a}$ NLR as a continuous variable

${ }^{\mathrm{b}} \mathrm{NLR}$ as a dichotomous variable

risk for the occurrence of HT after treatment with IV rtPA. Similarly, when we reintegrate the patients with infection or other conditions with potential to change the NLR into our analysis, the baseline NLR is higher in patients with HT (Additional file 1: Figure S3).

Moreover, the neutrophil and lymphocyte counts after ischemic stroke exhibit significant temporal variation [17], which is also indirectly confirmed by Maestrini et al. in their evaluation of the influence of the onset-tosample time on the neutrophil count, leukocyte count, and NLR [14]. This suggests that the neutrophil count, lymphocyte count, and NLR are "dynamic" variables. However, Maestrini et al. mainly focused on static neutrophil count and NLR values at baseline [14], which may not have dynamically and comprehensively reflected the patients' conditions. The clinical application value of dynamically testing the neutrophil count, lymphocyte count, and NLR in sICH and worse outcomes may be meaningful. Holding strict exclusion criteria in our study, we found that the NLR changed dynamically and a high NLR at 12-18 h after treatment with IV rtPA was independently associated with HT after IV rtPA. In addition, although there were no differences between the NLR at $12-18 \mathrm{~h}$ and the NLR at $36-48 \mathrm{~h}$ for auxiliary diagnosis of $\mathrm{PH}$ and $\mathrm{SICH}$, we hold that the NLR at 12$18 \mathrm{~h}$ may be more valuable than that at $36-48 \mathrm{~h}$. First, the NLR at $12-18 \mathrm{~h}$ could be obtained earlier than the NLR at 36-48 h, which could allow for better monitoring and could better reflect the severity and progression of disease, helping clinicians to adjust medication regimens and apply related auxiliary examination in time. Moreover, the NLR at $12-18 \mathrm{~h}$ was not inferior to the NLR at 36-48 h, and it exhibited a tendency to rise superior to the NLR at 36-48 $\mathrm{h}$ for diagnosing $\mathrm{sICH}$. Therefore, we believe that the NLR at $12-18 \mathrm{~h}$ is the appropriate selection based on the main concerns in the present study.

The mechanisms underlying these observations are not well established, but they seem to be related to the roles of neutrophils and lymphocytes in ischemic insult and the disruption of the BBB. Circulating neutrophils are recruited to the site of cerebral injury shortly after ischemia occurs and then further contribute to $\mathrm{BBB}$ disruption and tissue damage via a variety of mechanisms [18-20]. Neutrophils have been shown to be an important source of MMP-9, which may open the BBB inside the lumen of the blood vessel by acting directly on tight junction proteins or may be absorbed into endothelial cells and act on the basement membrane [2]. In a rat cerebral ischemia model, treatments preventing neutrophil infiltration reduced MMP-9 released in the brain [21]. Moreover, inhibition or depletion of neutrophils can reduce the $\mathrm{BBB}$ breakdown and the rate of $\mathrm{HT}$ in ischemic stroke [3, 22]. In contrast, when neutrophils are increased via lipopolysaccharide or granulocyte colony-stimulating factor administration, there is an increase in BBB disruption in a mice model [23] and an increase in MMP-9 and rtPA-related HT in a rat stroke model [4]. In humans with ischemic stroke, early neutrophilia is associated with larger infarct volumes [24], and MMP-9-positive neutrophil infiltration has also been associated with disruption of the BBB, basal lamina type IV collagen degradation, and HT [5]. Thus, neutrophils may mediate HT through neutrophil-derived MMPs in ischemic stroke. In addition to neutrophil-derived MMPs, other factors released from neutrophils after stroke [20], including ROS, myeloperoxidase, elastase, cathepsin G, proteinase 3 , cytokines, and chemokines, can also disrupt the neurovascular unit and ultimately result in increased BBB permeability and $\mathrm{HT}$ [17].

In addition to the mechanisms mediated by factors released from neutrophils, novel aspects of neutrophil biology may also contribute to ischemic brain injury. Recently, activated neutrophils have been described to form neutrophil extracellular traps (NETs), a web-like structure composed of DNA, histones, and specific granule proteins, such as neutrophil elastase and myeloperoxidase, in response to various stimuli [25]. Recent evidence indicates that a lack of NETs during myocardial and liver ischemia/ reperfusion (I/R) injury offers significant cardioprotective, hepatoprotective, and anti-inflammatory effects [26, 27]. Furthermore, extracellular chromatin and histones exacerbate cerebral I/R injury in mice [28]. These results suggest that NETs may play a role in BBB disruption and tissue damage. Further studies are needed to explore whether NETs have deleterious effects on HT. 
The lymphocyte counts might serve as an index for general health, influenced by acute physiologic stress [29]. Relative lymphopenia on the other hand reflects the cortisol-induced stress response and sympathetic tone [30], which can increase the production of proinflammatory cytokines that aggravate ischemic injury [31]. This means that low lymphocyte counts in patients with HT are not merely an initial response to severe stroke, but that lymphocytes may be actively involved in a protective mechanism in the ischemic brain. Experimental evidence suggests that specific subtypes of lymphocytes (namely, regulatory $\mathrm{T}$ cells) play key roles in abrogating the inflammatory response and are major cerebroprotective immunomodulators in acute stroke [32]. Our findings suggest that lower lymphocyte counts in patients with HT (data not shown) might have been the result of fewer regulatory $T$ cells being available to curtail the inflammatory response, thereby leading to greater tissue damage. However, other subtypes of lymphocytes (namely, proinflammatory lymphocytes) may have a deleterious effect on $I / R$ injury [33]. It is uncertain which subtype of lymphocytes has a dominant role in the pathophysiology of cerebral ischemia, and we demonstrated that a decrease in lymphocytes as a whole has a negative effect on HT. Further studies are also needed to elucidate the complex immunomodulatory interactions that occur after stroke.

The NLR reflects the balance between neutrophil and lymphocyte levels, which may be comprehensively represent the immunological conditions. In this sense, the NLR is superior to only the neutrophil count or lymphocyte count for distinguishing the occurrence of HT, and this may also explain why the AUC for the NLR appeared to be greater than those for neutrophil and lymphocyte counts at each corresponding time point (Additional file 1: Table S4). On the other hand, inflammatory cytokines released by neutrophils may trigger lymphocyte apoptosis [34]. This suggests that the NLR may not simply reflect the neutrophil and lymphocyte counts but also overactivation of neutrophils, thus leading to a wider gap between the two leukocyte types, and this also supports the superiority of the NLR.

The main strength of our study is that the clinical information and blood samples taken at different time points from all patients were collected in a prospective fashion with strict exclusion criteria. Previous infections and early hospital infections in stroke are associated with an increase in leukocytes and poor outcomes [14, 35]. We limited these potential confounders by ruling out patients with infection. In addition, our observations of elevated neutrophil counts in patients with HT (data not shown) may offer a partial explanation as to why stroke patients with infection may have poor outcomes. Nonetheless, our current findings also have some limitations.
First, the small sample size weakens the statistical strength of our conclusions. Therefore, further studies with larger samples are needed. Second, the study population included patients receiving a bridging strategy of the use of IV rtPA followed by endovascular therapy, which may interfere with our results. Nevertheless, similar results were found for patients experiencing a bridging strategy even if the sample was small (data not shown). On the other hand, this shows that the NLR may also be applicable to distinguish the presence and absence of HT in other reperfusion strategies such as endovascular therapy. Regrettably, a control group that did not experience reperfusion therapy was not included in our study. Third, we neither explored the mechanisms by which neutrophils and lymphocytes affect the BBB breakdown and HT nor investigated what factors regulate the dynamic changes in neutrophil and lymphocyte counts after ischemic stroke in animal studies. These will be the focus of our next work.

\section{Conclusions}

In conclusion, our results suggest that the NLR is a dynamic variable and its variation is associated with the occurrence of HT after thrombolysis in patients with stroke.

\section{Additional file}

Additional file 1: Tables S1 to S3 and Figures S1 to S4. (PDF $461 \mathrm{~kb}$ )

\section{Acknowledgements}

Not applicable.

Funding

This study was supported in part by the National Natural Science Foundation of China (Nos. 81171099, 81501193, and 81471182). The collection of this study was supported in part by the National Natural Science Foundation of China (Nos. 81171099, 81501193, and 81471182). The design of the study and analysis and interpretation of data and writing the manuscript was supported by the National Natural Science Foundation of China (Nos. 81171099 and 81501193).

Availability of data and materials

The datasets during and/or analyzed during the current study are available from the corresponding author on reasonable request.

\section{Authors' contributions}

ZLG was responsible for the study design, interpretation of the results, statistical analysis, and manuscript drafting. SHY and LLX were involved in the study design, interpretation of the results, statistical analysis, and critical revision of the manuscript. XC, CSZ, and SPW performed the data collection. RDY, QLD, and WS were involved in the study design and critical revision of the manuscript. PZ was in involved in the critical revision of the manuscript. WSZ and XFL participated in the study design, interpretation of the results, and critical revision of the manuscript. All authors read and approved the final manuscript.

Competing interests

The authors declare that they have no competing interests. 


\section{Consent for publication}

Not applicable.

\section{Ethics approval and consent to participate}

The study protocol was approved by the Institutional Human Research Ethics Committees of Jinling Hospital and PLA 123 Hospital, and all patients or their relatives gave informed consent.

\begin{abstract}
Author details
${ }^{1}$ Department of Neurology, Jinling Hospital, Medical School of Nanjing University, 305 E Zhongshan Rd, Nanjing 210002, Jiangsu Province, China. ${ }^{2}$ Department of Neurology, Second Affiliated Hospital of Soochow University, Suzhou 215004, China. ${ }^{3}$ Department of Medicine, Royal Melbourne Hospital, University of Melbourne, Melbourne, Australia. ${ }^{4}$ Department of Radiology, Jinling Hospital, Medical School of Nanjing University, Nanjing 210002, China. ${ }^{5}$ Department of Neurology, PLA 123 Hospital, 1052 Yanshan Road, Yuhui District, Bengbu 233000, China
\end{abstract}

\section{Received: 15 December 2015 Accepted: 18 August 2016} Published online: 26 August 2016

\section{References}

1. Emberson J, Lees KR, Lyden P, Blackwell L, Albers G, Bluhmki E, et al. Effect of treatment delay, age, and stroke severity on the effects of intravenous thrombolysis with alteplase for acute ischaemic stroke: a meta-analysis of individual patient data from randomised trials. Lancet. 2014;384:1929-35.

2. Jickling GC, Liu D, Stamova B, Ander BP, Zhan X, Lu A, et al. Hemorrhagic transformation after ischemic stroke in animals and humans. J Cereb Blood Flow Metab. 2014:34:185-99.

3. Gautier S, Ouk T, Petrault O, Caron J, Bordet R. Neutrophils contribute to intracerebral haemorrhages after treatment with recombinant tissue plasminogen activator following cerebral ischaemia. Br J Pharmacol. 2009; 156:673-9.

4. Gautier S, Ouk T, Tagzirt M, Lefebvre C, Laprais M, Petrault O, et al. Impact of the neutrophil response to granulocyte colony-stimulating factor on the risk of hemorrhage when used in combination with tissue plasminogen activator during the acute phase of experimental stroke. J Neuroinflammation. 2014;11:96.

5. Rosell A, Cuadrado E, Ortega-Aznar A, Hernandez-Guillamon M, Lo EH, Montaner J. MMP-9-positive neutrophil infiltration is associated to bloodbrain barrier breakdown and basal lamina type IV collagen degradation during hemorrhagic transformation after human ischemic stroke. Stroke. 2008;39:1121-6.

6. Gokhan S, Ozhasenekler A, Mansur Durgun H, Akil E, Ustundag M, Orak M. Neutrophil lymphocyte ratios in stroke subtypes and transient ischemic attack. Eur Rev Med Pharmacol Sci. 2013;17:653-7.

7. Tokgoz S, Keskin S, Kayrak M, Seyithanoglu A, Ogmegul A. Is neutrophil/ lymphocyte ratio predict to short-term mortality in acute cerebral infarct independently from infarct volume? J Stroke Cerebrovasc Dis. 2014;23:2163-8.

8. Brooks SD, Spears C, Cummings C, VanGilder RL, Stinehart KR, Gutmann L, et al. Admission neutrophil-lymphocyte ratio predicts 90 day outcome after endovascular stroke therapy. J Neurointerv Surg. 2014;6:578-83.

9. Montaner J, Molina CA, Monasterio J, Abilleira S, Arenillas JF, Ribo M, et al. Matrix metalloproteinase-9 pretreatment level predicts intracranial hemorrhagic complications after thrombolysis in human stroke. Circulation. 2003;107:598-603.

10. Hacke W, Kaste M, Fieschi C, von Kummer R, Davalos A, Meier D, et al. Randomised double-blind placebo-controlled trial of thrombolytic therapy with intravenous alteplase in acute ischaemic stroke (ECASS II). Second European-Australasian Acute Stroke Study Investigators. Lancet (London, England). 1998:352:1245-51.

11. Hacke W, Kaste M, Fieschi C, Toni D, Lesaffre E, von Kummer R, et al. Intravenous thrombolysis with recombinant tissue plasminogen activator for acute hemispheric stroke. The European Cooperative Acute Stroke Study (ecass). JAMA. 1995;274:1017-25.

12. DeLong ER, DeLong DM, Clarke-Pearson DL. Comparing the areas under two or more correlated receiver operating characteristic curves: a nonparametric approach. Biometrics. 1988;44:837-45.
13. Saliba W, Barnett-Griness O, Elias M, Rennert G. Neutrophil to lymphocyte ratio (NLR) and risk of first episode stroke in patients with atrial fibrillation: a cohort study. J Thromb Haemost. 2015;13:1971-9.

14. Maestrini I, Strbian D, Gautier S, Haapaniemi E, Moulin S, Sairanen T, et al. Higher neutrophil counts before thrombolysis for cerebral ischemia predict worse outcomes. Neurology. 2015;85:1408-16.

15. McColl BW, Allan SM, Rothwell NJ. Systemic infection, inflammation and acute ischemic stroke. Neuroscience. 2009;158:1049-61.

16. Dziedzic T. Systemic inflammation as a therapeutic target in acute ischemic stroke. Expert Rev Neurother. 2015;15:523-31.

17. Jickling GC, Liu D, Ander BP, Stamova B, Zhan X, Sharp FR. Targeting neutrophils in ischemic stroke: translational insights from experimental studies. J Cereb Blood Flow Metab. 2015;35:888-901.

18. Perez-de-Puig I, Miro-Mur F, Ferrer-Ferrer M, Gelpi E, Pedragosa J, Justicia C, et al. Neutrophil recruitment to the brain in mouse and human ischemic stroke. Acta Neuropathol. 2015;129:239-57.

19. Neumann J, Riek-Burchardt M, Herz J, Doeppner TR, Konig R, Hutten H, et al. Very-late-antigen-4 (VLA-4)-mediated brain invasion by neutrophils leads to interactions with microglia, increased ischemic injury and impaired behavior in experimental stroke. Acta Neuropathol. 2015;129:259-77.

20. Kolaczkowska E, Kubes P. Neutrophil recruitment and function in health and inflammation. Nat Rev Immunol. 2013:13:159-75.

21. Justicia C, Panes J, Sole S, Cervera A, Deulofeu R, Chamorro A, et al. Neutrophil infiltration increases matrix metalloproteinase-9 in the ischemic brain after occlusion/reperfusion of the middle cerebral artery in rats. J Cereb Blood Flow Metab. 2003;23:1430-40.

22. Zhang L, Zhang ZG, Zhang RL, Lu M, Krams M, Chopp M. Effects of a selective CD11b/CD18 antagonist and recombinant human tissue plasminogen activator treatment alone and in combination in a rat embolic model of stroke. Stroke. 2003;34:1790-5.

23. McColl BW, Rothwell NJ, Allan SM. Systemic inflammatory stimulus potentiates the acute phase and CXC chemokine responses to experimental stroke and exacerbates brain damage via interleukin-1- and neutrophildependent mechanisms. J Neurosci. 2007;27:4403-12.

24. Buck BH, Liebeskind DS, Saver JL, Bang OY, Yun SW, Starkman S, et al. Early neutrophilia is associated with volume of ischemic tissue in acute stroke. Stroke. 2008:39:355-60.

25. Brinkmann V, Reichard U, Goosmann C, Fauler B, Uhlemann Y, Weiss DS, et al. Neutrophil extracellular traps kill bacteria. Science. 2004;303:1532-5.

26. Savchenko AS, Borissoff Jl, Martinod K, De Meyer SF, Gallant M, Erpenbeck L, et al. WW-mediated leukocyte recruitment with chromatin decondensation by PAD4 increases myocardial ischemia/reperfusion injury in mice. Blood. 2014;123:141-8.

27. Huang H, Tohme S, Al-Khafaji AB, Tai S, Loughran P, Chen L, et al. Damageassociated molecular pattern-activated neutrophil extracellular trap exacerbates sterile inflammatory liver injury. Hepatology. 2015;62:600-14.

28. De Meyer SF, Suidan GL, Fuchs TA, Monestier M, Wagner DD. Extracellular chromatin is an important mediator of ischemic stroke in mice. Arterioscler Thromb Vasc Biol. 2012;32:1884-91.

29. Li Z, Cui L, Ma J, Ma X, Li G. Association between neutrophil to lymphocyte ratio and atrial fibrillation. Int J Cardiol. 2015;187:361-2.

30. Acanfora D, Gheorghiade M, Trojano L, Furgi G, Pasini E, Picone C, et al. Relative lymphocyte count: a prognostic indicator of mortality in elderly patients with congestive heart failure. Am Heart J. 2001;142:167-73.

31. Park BJ, Shim JY, Lee HR, Lee JH, Jung DH, Kim HB, et al. Relationship of neutrophil-lymphocyte ratio with arterial stiffness and coronary calcium score. Clin Chim Acta. 2011:412:925-9.

32. Liesz A, Suri-Payer E, Veltkamp C, Doerr H, Sommer C, Rivest S, et al. Regulatory $T$ cells are key cerebroprotective immunomodulators in acute experimental stroke. Nat Med. 2009;15:192-9.

33. Liesz $\mathrm{A}, \mathrm{Hu} \mathrm{X}$, Kleinschnitz $\mathrm{C}$, Offner $\mathrm{H}$. Functional role of regulatory lymphocytes in stroke: facts and controversies. Stroke. 2015;46:1422-30.

34. Shantsila E, Lip GY. Stroke in atrial fibrillation and improving the identification of 'high risk' patients: the crossroads of immunity and thrombosis. J Thromb Haemost. 2015;13:1968-70.

35. Tiainen M, Meretoja A, Strbian D, Suvanto J, Curtze S, Lindsberg PJ, et al. Body temperature, blood infection parameters, and outcome of thrombolysis-treated ischemic stroke patients. Int J Stroke. 2013:8:632-8. 